
\title{
BResarch Soutere \\ Effect of small photon field on dose distribution in transverse magnetic field: A Monte Carlo study
}

Jiaqi Fu

Shanghai Jiao Tong University

Cheng Ni

United Imaging Healthcare

Yanfang Liu

United Imaging Healthcare

Jingjie Zhou

United Imaging Healthcare

Jie Fu ( $\square$ fujie74@sjtu.edu.cn )

Shanghai Sixth Peoples Hospital

Original research

Keywords: MRI-Linac, EGSnrc, small photon field, dose distribution

Posted Date: February 25th, 2020

DOI: https://doi.org/10.21203/rs.2.24380/v1

License: (c) (i) This work is licensed under a Creative Commons Attribution 4.0 International License.

Read Full License 


\section{Effect of small photon field on dose distribution in transverse magnetic field: A Monte Carlo study}

Jiaqi Fu, MS ${ }^{1}$, Cheng Ni, $\mathrm{PhD}^{1,2}$, Yanfang Liu, $\mathrm{MS}^{2}$, Jingjie Zhou, $\mathrm{MS}^{2}$, Jie Fu, $\mathrm{PhD}^{3^{*}}$

${ }^{1}$ School of Biomedical Engineering, Shanghai JiaoTong University, Shanghai, China

${ }^{2}$ Radiotherapy Devision, United Imaging Healthcare, Shanghai, China

${ }^{3}$ Department of Radiotherapy, Shanghai Sixth People's Hospital Affiliated to Shanghai

JiaoTong University, Shanghai, China

${ }^{*}$ Corresponding author

Contact Information

Jie $\mathrm{Fu}$

Department of Radiotherapy

Shanghai Sixth People's Hospital Affiliated to Shanghai JiaoTong University

Shanghai 200000

China

Email: fujie@sjtu.edu.cn 


\section{Abstract}

Background: To investigate the effect of small photon field on dose distribution in transverse magnetic field by simulating the dose distribution on homogenous and heterogenous phantoms.

Methods: EGsnrc simulation was used to calculate the dose distribution of four phantoms irradiated by small photon field in transverse magnetic field $B=0 T, B=0.35 T$ and $B=1.5 T$. The photon beams were based on the phase space file of Varian Clinac_iX 6MV from the IAEA's online accelerator phase space database. The size of beams was $0.5 \mathrm{~cm} \times 0.5 \mathrm{~cm}, 1 \mathrm{~cm} \times 1 \mathrm{~cm}, 2 \mathrm{~cm} \times 2 \mathrm{~cm}$ and $4 \mathrm{~cm} \times 4 \mathrm{~cm}$, respectively.

Results: In homogenous water phantom and $1.5 \mathrm{~T}$ magnetic field, the distance of the dose buildup region decreased by $4 \mathrm{~mm}$ compared with that without magnetic field. At the edge of the water phantom, the dose of central axis changed a $1.7 \%$ decrease in the field of $0.5 \mathrm{~cm} \times 0.5 \mathrm{~cm}$ to a $27 \%$ increase in the field of $4 \mathrm{~cm} \times 4 \mathrm{~cm}$. The lateral profiles shifted by $1-3 \mathrm{~mm}$ and the dose penumbra had asymmetry. In $0.35 \mathrm{~T}$ magnetic field, the effect of magnetic field was insignificant.

In water-air and water-lung phantom, the dose at the water-air and water-lung interface increased by $24.2 \%, 30.9 \%$, respectively. However, at the air-water and lung-water interface, it decreased by $10.9 \%$ and $29.9 \%$ in the field of $2 \mathrm{~cm} \times 2 \mathrm{~cm}$. In $0.35 \mathrm{~T}$ magnetic field, there was no significant change in the dose at the interface. In water-bone phantom, the dose at the water-bone interface increased.

Conclusions: In 1.5T magnetic field, when the field was small, the shift of lateral profile was obvious and the dose penumbra had asymmetry. At the interface of different tissue, the dose change depends on the size of the field, the strength of magnetic field and the change of tissue density. The effect of the magnetic field on the dose distribution needs to be considered during optimizing a treatment plan, to avoid dose hotspots at normal tissues. 
Key words: MRI-Linac EGSnrc small photon field dose distribution

\section{Background}

Radiotherapy is a common and important method for cancer treatment. Currently, more than $50 \%$ of cancer patients need radiation therapy (1). In recent years, with the rapid development of physical technology in radiotherapy, image-guided radiotherapy (IGRT) technology obtained more and more applications. By means of image guidance, the tumor and surrounding normal organs can be monitored before and after treatment, and even during treatment. Clinically mainstream devices for IGRT use X-ray imaging, but they are difficult to monitor the movement of the tumor during treatment. MRI-guided radiotherapy not only significantly improves the definition of target areas and reduces motion artifacts, but also monitors tumor movements in real-time during treatment and optimizs treatment plans online. Also, it can avoid extra ionizing radiation.

At present, four research institutes are engaging in integrating MRI and linear accelerator (MRILinac). Clinically, making magnetic field perpendicular to the photon beam is the main configuration of MRI-Linac. The MRI-Linacs system of ViewRay firstly integrated a 0.35 T MRI with three ${ }^{60} \mathrm{CO}$ radioactive sources, and then the three ${ }^{60} \mathrm{CO}$ were replaced by a $6 \mathrm{MV}$ Linear accelerator (2); Elekta's MRI-Linac system integrated a 1.5T MRI with a 7MV linear accelerator (3).

In recent years, modern treatment mode of high precision, high dose, high efficacy and low damage was realized, bringing the emerging of high-precision radiotherapy such as stereotactic body radiotherapy (SBRT). Compared with conventional radiotherapy, SBRT has the advantages of 
more precise positioning, lower exposure dose to normal organ and higher exposure dose to tumor target (4). There are more and more clinical studies on the application of SBRT in locally advanced cancer and advanced oligometastases (5). Intensity-modulated radiotherapy, volumetric rotational intensity-modulated radiotherapy and spiral tomotherapy are the main technologies of SBRT. In modern high-precision radiotherapy technology, it involves the application of smal fields.

Lung cancer is one of the leading fatal cancers in the world. Surgical resection is the main treatment. The development of SBRT provides a new treatment option for patients who are not surgically resectable, and becomes the main choice for patients with stage I unresectable NSCLC (6). A series of prospective studies have shown a local control rate of approximately $90 \%$, comparable to limited resection surgery (7-8). MRI-Linac can monitor tumor movement in real time during treatment, such that doctors and physicistss can reduce the planning target volume (PTV) margins and optimize daily planning based on the daily MRI (9). This promotes the application of SBRT technology in the treatment of lung cancer, increases the dose of the target and decreases the treatment time under the premise of protecting the crisis organs.

However, charged particles (eg, electrons) transporting in the magnetic field are affected by the Lorentz force, and the motion trajectory is deflected. At the boundary where the tissue density varies greatly (eg, water-air, water-lung), the electrons are deflected back into the high density region from low density region due to the action of the magnetic field, which is called the electron return effect (ERE) (10). Many studies have shown that the effect of transverse magnetic field on dose disturbance was mainly reflected in the ERE, the lateral shift of the dose distribution and the penumbra asymmetry vertical along the direction of the magnetic field, and the distance reduction of the dose build-up region (11-17). These studies are mainly for the photon beam field of 
$5 \mathrm{~cm} \times 5 \mathrm{~cm}$ or above. The results of Raaymakers et al. showed that in the $1.5 \mathrm{~T}$ magnetic field, the distance between the dose build-up region in the water phantom reduced by $5 \mathrm{~mm}$, and the penumbra shifted $0.7 \mathrm{~mm}$ when the field was $5 \mathrm{~cm} \times 5 \mathrm{~cm}$ (11). Sebastian Richter et al. used a $20 \times 20 \times 20 \mathrm{~cm}^{3}$ water phantom and a $10 \times 10 \times 10 \mathrm{~cm}^{3}$ water-air phantom to study the dose characteristics of $1 \mathrm{~cm} \times 1 \mathrm{~cm}, 4 \mathrm{~cm} \times 4 \mathrm{~cm}, 5 \mathrm{~cm} \times 5 \mathrm{~cm}$ fields in different magnetic fields. The results showed that the dose penumbra in the water phantom shifted by $3 \mathrm{~mm}$ in a $1.5 \mathrm{~T}$ magnetic field (17). Compared with the results of Raaymakers, the dose penumbra shift was more obvious, which may due to the effect of magnetic field on electron equilibrium in the small field.

This presented work investigating the effect of magnetic fields on the dose distribution of small field $(1 \mathrm{~cm} \times 1 \mathrm{~cm}, 2 \mathrm{~cm} \times 2 \mathrm{~cm}$, and $4 \mathrm{~cm} \times 4 \mathrm{~cm})$ and extremely small field $(0.5 \mathrm{~cm} \times 0.5 \mathrm{~cm})$. The $0.35 \mathrm{~T}$ and $1.5 \mathrm{~T}$ magnetic fields were used to study the effect of magnetic field, which was the magnetic field strength of clinical MRI-Linac. EGSnrc was used to simulate the distribution of doses in four different phantoms, and the effect of magnetic field on dose characteristics in different phantoms was analyzed.

\section{Methods}

\subsection{Monte Carlo simulation}

The EGSnrc Monte Carlo code system was used for all presented simulation. The enhanced electric and magnetic field (EEMF) implemented by Malkov was used to study the influence of magnetic field (18). Beams were based on the phase space file of Varian Clinac_iX 6MV from the IAEA's online accelerator phase space database (19). Considering that this paper studied the influence of the magnetic field on the dose distribution in the small field, this work only selected the phase space file which the size of field below $5 \mathrm{~cm} \times 5 \mathrm{~cm}$. Four phantom geometries were 
modelled to investigate the effect of magnetic field on different tissue's interface.

All phantoms were $12 \times 12 \times 12 \mathrm{~cm}^{3}$ with a cubic voxel size of $1 \mathrm{~mm}^{3}$. The first phantom was a $12 \times 12 \times 12 \mathrm{~cm}^{3}$ water phantom irradiated by small filed and extremely small field. To investigate the effect of magnetic field on percentage depth dose (PDD) profiles and lateral profiles and compare the difference of the influence of magnetic field on the dose distribution of small field. The remaining three phantoms were water-air, water-lung and water-bone phantom, which were obtained by inserting $3 \mathrm{~cm}$ of air, lungs, and bone into the water phantom at a distance of $3 \mathrm{~cm}$ from the beam entrance respectively. These three phantoms were used to study the effect of magnetic field on the dose distribution of small photon beam at different tissue interfaces. During the Dosxyznrc simulation, density of water, air, lung, and bone were set to $1 \mathrm{~g} / \mathrm{cm}^{3}, 0.0039 \mathrm{~g} / \mathrm{cm}^{3}$, $0.39 \mathrm{~g} / \mathrm{cm}^{3}$, and $1.85 \mathrm{~g} / \mathrm{cm}^{3}$, respectively.

To investigate the effect of magnetic field, the EEMF was evoked during the electron transport to correct the trajectory of the electron in the magnetic field. Magnetic fields of 0T, 0.35T and 1.5T were defined over the entire geometry and the magnetic fields direction along the -y-direction and all beams were orientated in z-direction. In order to ensure the uncertainty of dose calculation, the number of simulated particles was $1 \times 10^{9}$. In order to visually compare the difference in dose distribution, all doses were normalized by the the maximum dose of $\mathrm{B}=0 \mathrm{~T}$ at the same phantom and the same beam field.

\section{Results}

\section{1 water phantom}

The PDD and lateral profile characteristics in the magnetic field were studied by a uniform water 
phantom irradiated by small field. The results in Figure 1 showed the PDD curves of different sizes of field at $0.35 \mathrm{~T}$ and $1.5 \mathrm{~T}$ magnetic fields respectively. The maximum dose in 0T magnetic field was used as the reference dose. As depicted in Figure1 (b), in the 1.5T magnetic field, when the field was $2 \mathrm{~cm} \times 2 \mathrm{~cm}$ and $4 \mathrm{~cm} \times 4 \mathrm{~cm}$, the distance between the dose build-up region decreased by $4 \mathrm{~mm}$, the maximum dose on the central axis increased by $2.4 \%$ and $1 \%$ respectively. When the field was $1 \mathrm{~cm} \times 1 \mathrm{~cm}$ and $0.5 \mathrm{~cm} \times 0.5 \mathrm{~cm}$, the build-up region distance only decreased by 1 $2 \mathrm{~mm}$, and the maximum dose on the central axis reduced by $3 \%$. As the size of field increased, the dose of the central axis at the end of phantom increased from $-1.7 \%(0.5 \mathrm{~cm} \times 0.5 \mathrm{~cm})$ to $27 \%$ $(4 \mathrm{~cm} \times 4 \mathrm{~cm})$ due to the influence of the ERE. At 0.35T, the effect of the magnetic field on PDD was almost negligible, and the end of phantom's dose was almost no increase.

Figure 2-3 showed the lateral profiles at a depth of the maximum dose. From the results shown, the lateral dose curve of the beam shifted and the dose penumbra was asymmetrical due to the Lorentz force. Since the direction of the magnetic field was along the y-axis, the lateral dose curve in the $\mathrm{Y}$ direction hardly shifted, and only the lateral dose curve in the $\mathrm{X}$ direction shifted to a different extent, and the penumbra in the offset direction became steeper. In the 1.5T magnetic field, the curves offset by about $1-3 \mathrm{~mm}$. The center dose of the lateral curves of the $2 \mathrm{~cm} \times 2 \mathrm{~cm}$ and $4 \mathrm{~cm} \times 4 \mathrm{~cm}$ fields was almost the same as those without magnetic field. While the center dose increased $1 \%$ in the $1 \mathrm{~cm} \times 1 \mathrm{~cm}$ field and decreased $5 \%$ in the $0.5 \mathrm{~cm} \times 0.5 \mathrm{~cm}$ field. In the $0.35 \mathrm{~T}$ magnetic field, there was almost no offset in the lateral curve. In the $2 \mathrm{~cm} \times 2 \mathrm{~cm}$ field, an offset of about $1 \mathrm{~mm}$ occurred in the profile. There was no significant difference between the dose with magnetic field and the dose without the magnetic field.

\section{2 water-air and water-lung phantom}


Water-air phantom and water-lung phantom were mainly used to study the effect of ERE on the dose distribution at the interface. The average free path length of electrons in the lungs and air is greater than the electron deflection radius, thus the electrons were returned by Lorentz force and went back to the high-density area from low density. PDDs in Figure 4-5 showed that the dose with the magnetic field in the air or lung region was lower than the dose without the magnetic field. When the field size was $2 \mathrm{~cm} \times 2 \mathrm{~cm}$ and $4 \mathrm{~cm} \times 4 \mathrm{~cm}$, the dose at the water-lung and water-air interface increased, and a dose reduction occured at the lung-water and air-water interfaces and after the beam passed through the air or lungs, re-appearing the dose build-up region. In the 1.5T magnetic field, the water-air phantom increased the dose by $24.2 \%$ and $38.1 \%$ at the water-air interface $z=29 \mathrm{~mm}$, respectively. The dose decreased by $10.9 \%$ and $29.9 \%$ at the air-water interface $z=60 \mathrm{~mm}$; In the lung phantom, the dose was increased by $30.9 \%$ and $36.4 \%$ at $z=31 \mathrm{~mm}$ at the water-lung interface, respectively, and the dose decreased by $21.3 \%$ and $24.7 \%$ at the lung-water interface $z=$ $60 \mathrm{~mm}$, respectively. However, in the $0.35 \mathrm{~T}$ magnetic field, there was a strange phenomenon. When the size of field was $2 \mathrm{~cm} \times 2 \mathrm{~cm}$, the dose with the magnetic field was higher than the dose without the magnetic field before entering the air or the lung $1 \mathrm{~cm}$, and in the second $2 \mathrm{~cm}$, the dose with the magnetic field was less than the dose without the magnetic field. Thus the dose at the air-water and water-lung interface reduced by $8 \%$ and $5 \%$, respectively. When the field size was $1 \mathrm{~cm} \times 1 \mathrm{~cm}$ and $0.5 \mathrm{~cm} \times 0.5 \mathrm{~cm}$, there was no obvious dose change at the interface, but it was worthnoting that the dose on the central axis in the $1.5 \mathrm{~T}$ magnetic field will be lower than the dose without the magnetic field. In $0.35 \mathrm{~T}$ magnetic field, there was no significant change in the dose at the interface.

\section{3 water-bone phantom}

Bone is a high-density tissue. The water-bone phantom was mainly used to study the effect of 
magnetic field on the dose distribution in high-density areas. The PDD characteristics in the water phantom on both sides of the bone tissue were consistent with the PDD characteristics of the region in the homogenous water phantom, water-air phantom and water-lung phantom studied previously.

As was shown in the PDD curves of Figure 6, the slope of the curve in the bone tissue was greater than that in the water. Because the bone tissue density is greater than the density of the water, the energy deposited in the bone tissue is also greater. In 0T magnetic field, when the size of the field was $2 \mathrm{~cm} \times 2 \mathrm{~cm}$ and $4 \mathrm{~cm} \times 4 \mathrm{~cm}$, the local maximum dose point appeared at the water-bone interface $z=29 \mathrm{~mm}$, however, when the field size was $1 \mathrm{~cm} \times 1 \mathrm{~cm}$ and $0.5 \mathrm{~cm} \times 0.5 \mathrm{~cm}$, the local maximum dose appeared at $z=29 \mathrm{~mm}$ and $z=33 \mathrm{~mm}$, respectively. The difference between PDDs in 1.5T magnetic field and PDDs in 0T magnetic field was mainly reflected at the water-bone and bone-water interface. In the $1.5 \mathrm{~T}$ magnetic field, the local maximum dose points at the water-bone interface in $4 \mathrm{~cm} \times 4 \mathrm{~cm}, 2 \mathrm{~cm} \times 2 \mathrm{~cm}$, and $1 \mathrm{~cm} \times 1 \mathrm{~cm}$ fields appeared at $z=$ $35 \mathrm{~mm}$ and $\mathrm{z}=34 \mathrm{~mm}$ in the field of $0.5 \mathrm{~cm} \times 0.5 \mathrm{~cm}$. When the field was greater than $0.5 \mathrm{~cm} \times 0.5 \mathrm{~cm}$, the same phenomenon occurs at the bone-water interface $z=61 \mathrm{~mm}$. The characteristics of the dose curve in the $0.35 \mathrm{~T}$ magnetic field were almost the same as those without magnetic field.

\section{Discussion}

Sebastian Richter et al. studied the dose distribution of $1 \mathrm{~cm} \times 1 \mathrm{~cm}$ and $4 \mathrm{~cm} \times 4 \mathrm{~cm}$ photon filed in different magnetic field (17). In this work, the dose curve characteristics of the $1 \mathrm{~cm} \times 1 \mathrm{~cm}$ and $4 \mathrm{~cm} \times 4 \mathrm{~cm}$ fields in the homogenous water phantom and the water-air phantom were consistent with the results of Sebastian Richter et al. In order to better study the dose curve characteristics of the small field in the magnetic field, $0.5 \mathrm{~cm} \times 0.5 \mathrm{~cm}$ and $2 \mathrm{~cm} \times 2 \mathrm{~cm}$ fields 
were also used in thiswork, and their characteristics in water-lung and water-bone phantoms were also studied.

In section 3.1, compared with the PDD curve of the 0T magnetic field, the build-up region of the dose was shortened in the $1.5 \mathrm{~T}$ magnetic field. As the size of field increased, the more contaminated electrons entered the phantom and the electrons were deflected to the entrance of the field due to the Lorentz force in the $1.5 \mathrm{~T}$ magnetic field. Resulting in the distance of the build-up region was shortened by $1-2 \mathrm{~mm}$ in $0.5 \mathrm{~cm} \times 0.5 \mathrm{~cm}$ field and $4 \mathrm{~mm}$ in $4 \mathrm{~cm} \times 4 \mathrm{~cm}$ field. In the 1.5T magnetic field. In the $2 \mathrm{~cm} \times 2 \mathrm{~cm}$ and $4 \mathrm{~cm} \times 4 \mathrm{~cm}$ fields, the maximum dose was increased by $1 \%-2.4 \%$ relative to the maximum dose of the $0 \mathrm{~T}$ magnetic field, but interestingly, the maximum dose relatively decreased in the $1 \mathrm{~cm} \times 1 \mathrm{~cm}$ and $0.5 \mathrm{~cm} \times 0.5 \mathrm{~cm}$ fields by $3 \%$, because the electron equilibrium in the magnetic field was damaged when the field is small, and the symmetry axis of the dose distribution shifted at $1.5 \mathrm{~T}$ magnetic field. This finding was also again proven by the lateral profile of $1 \mathrm{~cm} \times 1 \mathrm{~cm}$ and $0.5 \mathrm{~cm} \times 0.5 \mathrm{~cm}$ fields in $1.5 \mathrm{~T}$.

The dose increased at the end of the phantom due to the Lorentz force, which caused the electrons exiting the phantom to return to the phantom. The dose deposition at the end of the phantom increased. As the field increased, the dose deposition caused by the ERE increased. When the field was $0.5 \mathrm{~cm} \times 0.5 \mathrm{~cm}$, there was almost no dose increase at the end of the phantom in the magnetic field. This was because in $0.5 \mathrm{~cm} \times 0.5 \mathrm{~cm}$ field, after the beam passed through the $12 \mathrm{~cm}$ water phantom, the remaining energy was extremely low, and the emitted electrons were difficult to return to the phantom. The previous studies on the lateral profile curve were mainly in the case of greater than $4 \mathrm{~cm} \times 4 \mathrm{~cm}$ fields, and the results showed that the penumbra of the lateral profile would shift $0.7 \mathrm{~mm}$ (11). However, the study found that in the field of $4 \mathrm{~cm} \times 4 \mathrm{~cm}$ and below, the penumbra 
of the lateral profile curve in the vertical magnetic field was offset by $3 \mathrm{~mm}$, which was much larger than $0.7 \mathrm{~mm}$ when the field was more than $4 \mathrm{~cm} \times 4 \mathrm{~cm}$. In the treatment planning process, this phenomenon needs to be fully considered. In the $0.35 \mathrm{~T}$ magnetic field, there was almost no difference in the dose curve characteristics, compared with those in the absence of magnetic field.

In sections 3.2 and 3.3, the curves for the PDD of water-air, water-lung and water-bone phantoms were explained. In different tissues, the mean free path of electrons and the probability of interaction type with tissues were different. The smaller the density, the longer the mean free path of electrons. In the magnetic field, the electrons were deflected by the Lorentz force. The deflection radius was proportional to the electron energy and inversely proportional to the strength of the magnetic field. Studies have shown that when the deflection radius was larger than the size of the field, the electron equilibrium did not exist and the center of the dose lateral profile shifted [14]. This explained that the profile curve of the field at $1 \mathrm{~cm} \times 1 \mathrm{~cm}$ and $0.5 \mathrm{~cm} \times 0.5 \mathrm{~cm}$ in 3.1 not only shifted at the center, but also made the left and right penumbras asymmetrical in the $1.5 \mathrm{~T}$ magnetic field. In the magnetic field, when electrons enter the low-density area from the high-density area (such as waterair, water-lung interface), the mean free path of electrons becomes longer, and the electrons lose energy during the deflection process, and more electrons returned to the high density zone resulted the dose increased at the boundary. For case of $2 \mathrm{~cm} \times 2 \mathrm{~cm}$ and $4 \mathrm{~cm} \times 4 \mathrm{~cm}$ in 3.2, the dose at the water-lung and water-air interface increased, and there was no dose increase at the boundary between $1 \mathrm{~cm} \times 1 \mathrm{~cm}$ and $0.5 \mathrm{~cm} \times 0.5 \mathrm{~cm}$, mainly due to the shift of the dose center. The electron return effect reduced electrons entering the low density region, and the dose in the low density region in the presence of a magnetic field was significantly decreased relative to the dose in the absence of magnetic field, resulting in a decrease in dose at the air-water and air-lung interfaces. 
After the beam passes through the low-density area, it reaches equilibrium again and the second build-up region appears.

The PDD characteristics of the high-density region were studied in the water-bone phantom. Because the density of the bone was greater than the density of water, a small build-up region appeared at the water-bone interface. The depth of the small build-up region with the magnetic field was greater than that without magnetic field. The work of Ahmad showed that less scattered electrons in the magnetic field can reach the interface of the tissue, and electrons entering the highdensity region were more difficult to return to the low-density region (20). It caused that the distance between the build-up regions at the water-bone interface in the magnetic field was deeper than that without this magnetic field. In the $0.35 \mathrm{~T}$ magnetic field, because the electron radius of rotation was smaller, the degree of electron deflection was smaller, and the PDDs were almost indistinguishable from those without magnetic field.

\section{Conclusion}

This work mainly studied the dose curve characteristics of small field in homogenous water phantom and hetergenous water phantom in the magnetic field of $0.35 \mathrm{~T}$ and $1.5 \mathrm{~T}$. Compared with the dose curve characteristics in the case of non-small field, the $1.5 \mathrm{~T}$ magnetic field had a more obvious effect on the distribution of the small field, especially in the center offset of the lateral dose curve and the symmetry of the penumbra. Irradiated by the small field, the center offset increased to $3 \mathrm{~mm}$ from $0.7 \mathrm{~mm}$, and the penumbra asymmetry was more obvious. These characteristics must be fully considered in the development of treatment plans. In the $0.35 \mathrm{~T}$ magnetic field, the influence of the magnetic field on the dose distribution of small field was negligible.

Our work only studied the influence of magnetic field on the dose distribution of small field in 
phantoms. How to compensate the influence of the magnetic field on the dose distribution of small field in the treatment plan with the real case data is in our future research plan.

\section{List of abbreviations}

IGRT: image-guided radiotherapy

SBRT: stereotactic body radiotherapy

PTV: planning target volume

ERE: electron return effect

EEMF: enhanced electric and magnetic field

PDD: percentage depth dose

\section{Declarations}

Ethics approval and consent to participate: No applicable

Consent for publication: No applicable

Availability of data and materials: All data generated or analysed during this study are included in this published article [and its supplementary information files].

Competing interests: The authors declare that they have no competing interests

Funding: No applicable

Authors' contributions: Jiaqi Fu, Yanfang Liu and Jingjie Zhou designed the work and analyzed and interpreted the data. Cheng $\mathrm{Ni}$ and Jie Fu provided guidance on this work and revised the manuscript. All authors read and approved the final manuscript.

Acknowledgements: No applicable 


\section{Reference}

1. Peter B, Bernard L, et al. World cancer report 2008[R]. International Agency for Research on Cancer 2008. Britain. 2008.

2. Mutic S, Dempsey J F. The ViewRay system: magnetic resonance-guided and controlled radiotherapy[J]. Seminars in Radiation Oncology, 2014, 24(3):196-199.

3. Raaymakers B W, Lagendijk J J W, Overweg J, et al. Integrating a 1.5 T MRI scanner with a 6 MV accelerator: proof of concept[J]. Physics in Medicine and Biology, 2009, 54(12):N229---N237.

4. Taremi M, Hope A, Dahele M, et al. Stereotactic body radiotherapy for medically inoperable lung cancer: prospective, single-center study of 108 consecutive patients[J]. International Journal of Radiation Oncology Biology Physics, 2012, 82(2):967-973.

5. Rowe B P, Boffa D J, Wilson L D, et al. Stereotactic body radiotherapy for central lung tumors[J]. Journal of Thoracic Oncology, 2012, 7(9):1394-1399.

6. Timmerman, Robert. Stereotactic Body Radiation Therapy for Inoperable Early Stage Lung Cancer[J]. JAMA, 2010, 303(11):1070.

7. Williams F M, Mcgarry R, Timmerman R, et al. Extracranial stereotactic radioablation: results of a phase I study in medically inoperable stage I non-small cell lung cancer. [J]. Chest, 2003, 57(2):S280-S281.

8. Nagata Y, Takayama K, Matsuo Y, et al. Clinical outcomes of a phase I/II study of 48 Gy of stereotactic body radiotherapy in 4 fractions for primary lung cancer using a stereotactic body frame[J]. International Journal of Radiation Oncology Biology Physics, 2005, 63(5):1427-1431.

9. Kupelian P, Sonke J J. Magnetic Resonance-Guided Adaptive Radiotherapy: A Solution to the Future[J]. Seminars in Radiation Oncology, 2014, 24(3):227-232.

10. Raaijmakers A J E, Raaymakers B W, Lagendijk J J W. Magnetic-field-induced dose effects in MR-guided 
radiotherapy systems: dependence on the magnetic field strength[J]. Physics in Medicine and Biology, 2008, 53(4).

11. Raaymakers B W, Raaijmakers A J E, Kotte A N T J, et al. Integrating a MRI scanner with a 6 MV radiotherapy accelerator: dose deposition in a transverse magnetic field[J]. Physics in Medicine and Biology, 2004, 49(17):4109-4118.

12. Oborn B M, Metcalfe P E, Butson M J, et al. High resolution entry and exit Monte Carlo dose calculations from a linear accelerator $6 \mathrm{MV}$ beam under the influence of transverse magnetic fields[J]. Medical Physics, 2009, 36(8):3549.

13. Raaijmakers A J E, Raaymakers B W, Meer S V D, et al. Integrating a MRI scanner with a 6 MV radiotherapy accelerator: impact of the surface orientation on the entrance and exit dose due to the transverse magnetic field[J]. Physics in Medicine and Biology, 2007, 52(4):929-939.

14. Oborn B M, Metcalfe P E, Butson M J, et al. Monte Carlo characterization of skin doses in 6 MV transverse field MRI-linac systems: Effect of field size, surface orientation, magnetic field strength, and exit bolus[J]. Medical Physics, 2010, 37(10):5208.

15. Keyvanloo A, Burke B, Warkentin B, et al. Skin dose in longitudinal and transverse linac-MRIs using Monte Carlo and realistic 3D MRI field models[J]. Medical Physics, 2012, 39(10):6509.

16. Kirkby C, Stanescu T, Rathee S, et al. Patient dosimetry for hybrid MRI-radiotherapy systems[J]. Medical Physics, 2008, 35(3):1019.

17. Richter S, Pojtinger S, Mönnich D, et al. Influence of a transverse magnetic field on the dose deposited by a 6 MV linear accelerator[J]. Current Directions in Biomedical Engineering, 3(2).

18. Malkov V N, Rogers D W O. Charged particle transport in magnetic fields in EGSnrc[J]. Medical Physics, 2016, 43(7):4447-4458. 
19. www-nds.iaea.org/phsp/phsp.htmlx

20. Ahmad S B, Sarfehnia A, Kim A, et al. Backscatter dose effects for high atomic number materials being irradiated in the presence of a magnetic field: A Monte Carlo study for the MRI linac[J]. Medical Physics, 2016, 43(8):4665-4673. 


\section{Figure legends}

Figure 1. The PDD curves of water phantom in different size of beam field with magnetic field compared with those without magnetic field. (a) $\mathrm{B}=0.35 \mathrm{~T}$. (b) $\mathrm{B}=1.5 \mathrm{~T}$

Figure 2. The dose profiles of water phantom in different size of beam field with magnetic field $\mathrm{B}=0.35 \mathrm{~T}$ compared with those without magnetic field at the depth of the maximum dose. (a) Dose profiles in the $\mathrm{X}$ direction. (b) Dose profiles in the $\mathrm{Y}$ direction

Figure 3. The dose profiles of water phantom in different size of beam field with magnetic field $\mathrm{B}=1.5 \mathrm{~T}$ compared with those without magnetic field at the depth of the maximum dose. (a) Dose profiles in the $\mathrm{X}$ direction. (b) Dose profiles in the $\mathrm{Y}$ direction

Figure 4. The PDD curves of water-air phantom in different size of beam field with magnetic field compared with those without magnetic field. (a) $\mathrm{B}=0.35 \mathrm{~T}$. (b) $\mathrm{B}=1.5 \mathrm{~T}$

Figure 5. The PDD curves of water-lung phantom in different size of beam field with magnetic field compared with those without magnetic field. (a) $\mathrm{B}=0.35 \mathrm{~T}$. (b) $\mathrm{B}=1.5 \mathrm{~T}$

Figure 6. The PDD curves of water-bone phantom in different size of beam field with magnetic field compared with those without magnetic field. (a) $\mathrm{B}=0.35 \mathrm{~T}$. (b) $\mathrm{B}=1.5 \mathrm{~T}$ 
Figures

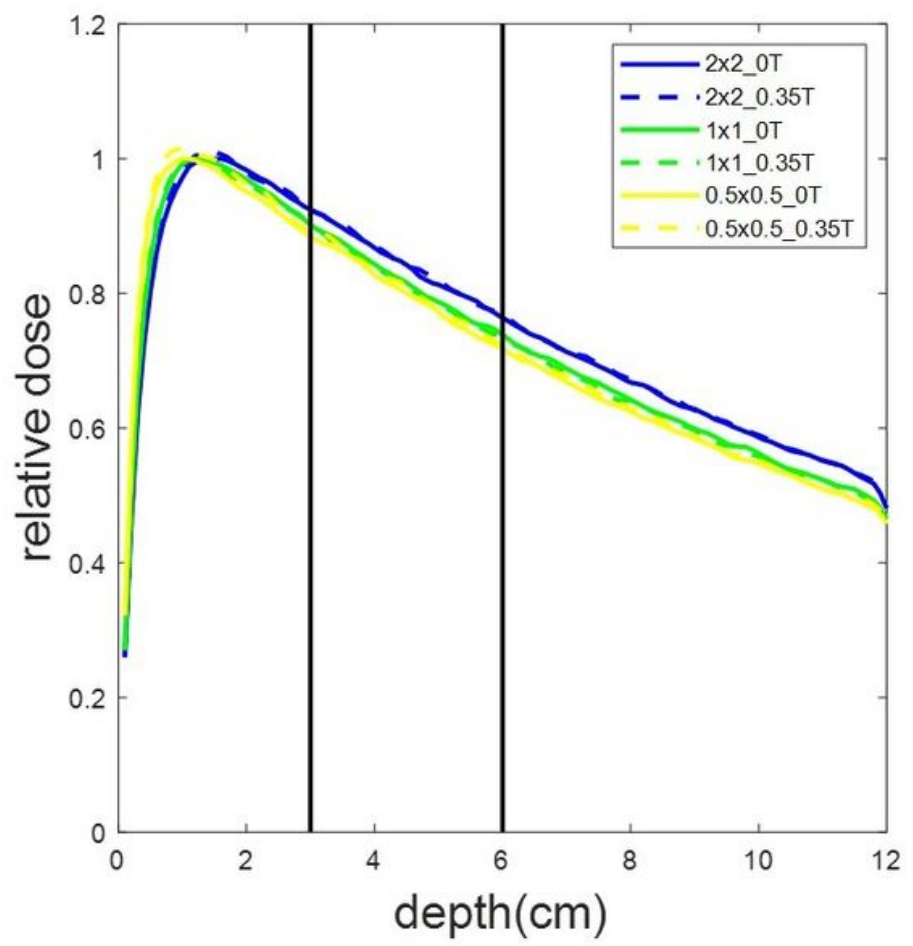

(a)

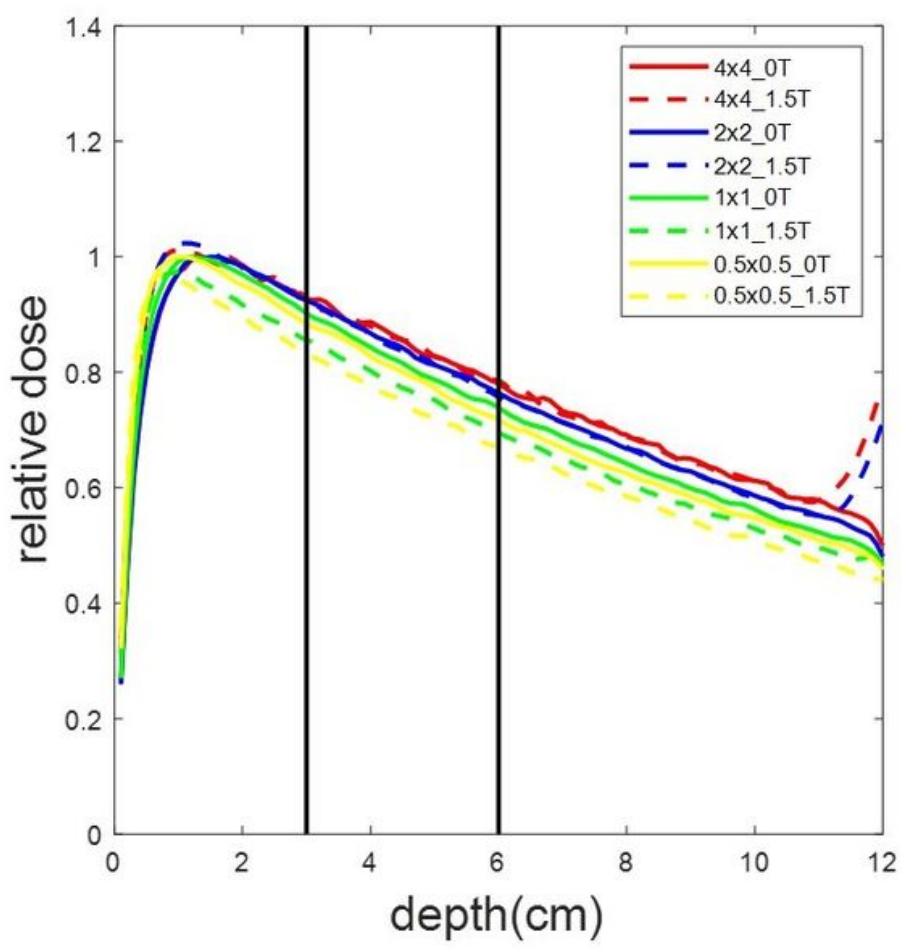

(b)

\section{Figure 1}

The PDD curves of water phantom in different size of beam field with magnetic field compared with those without magnetic field. (a) $B=0.35 \mathrm{~T}$. (b) $B=1.5 \mathrm{~T}$ 


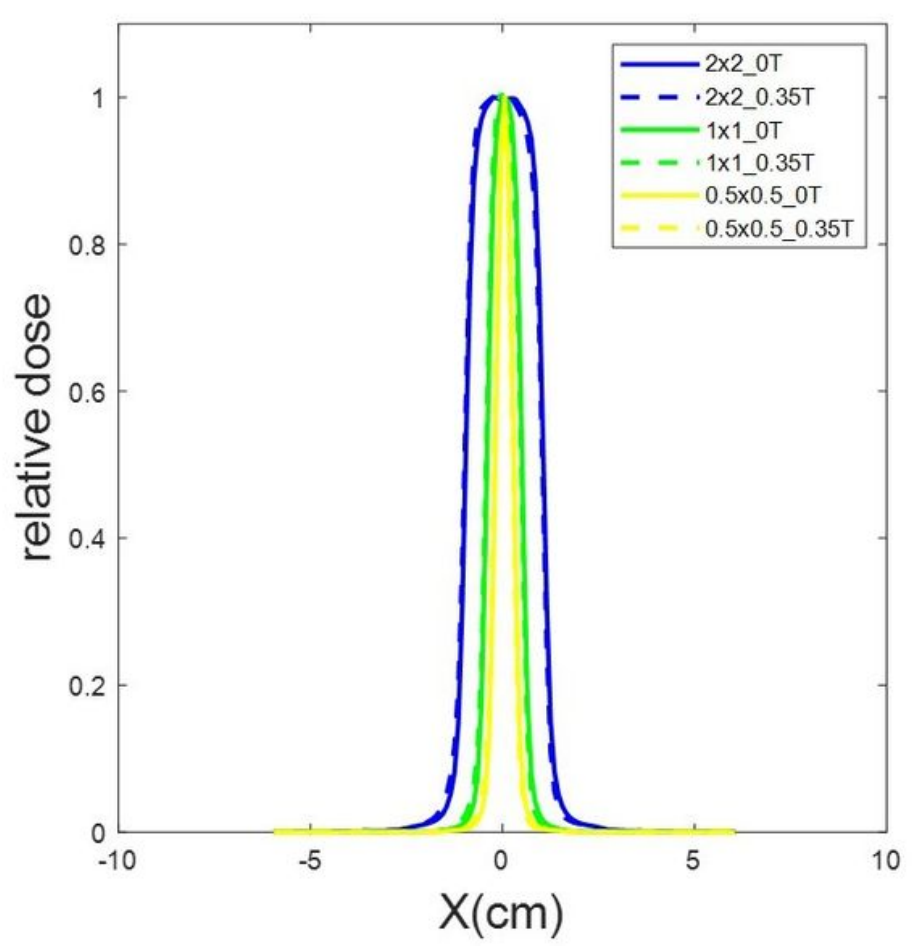

(a)

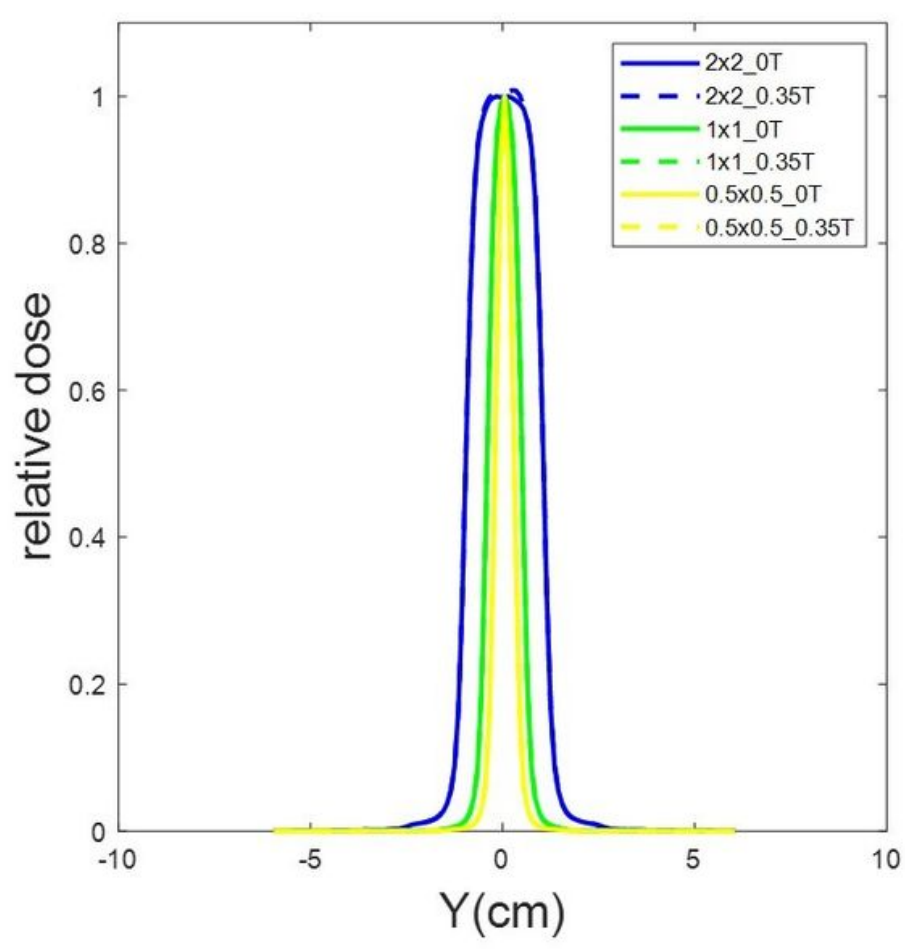

(b)

Figure 2

The dose profiles of water phantom in different size of beam field with magnetic field $B=0.35 T$ compared with those without magnetic field at the depth of the maximum dose. (a) Dose profiles in the $X$ direction.

(b) Dose profiles in the $Y$ direction 


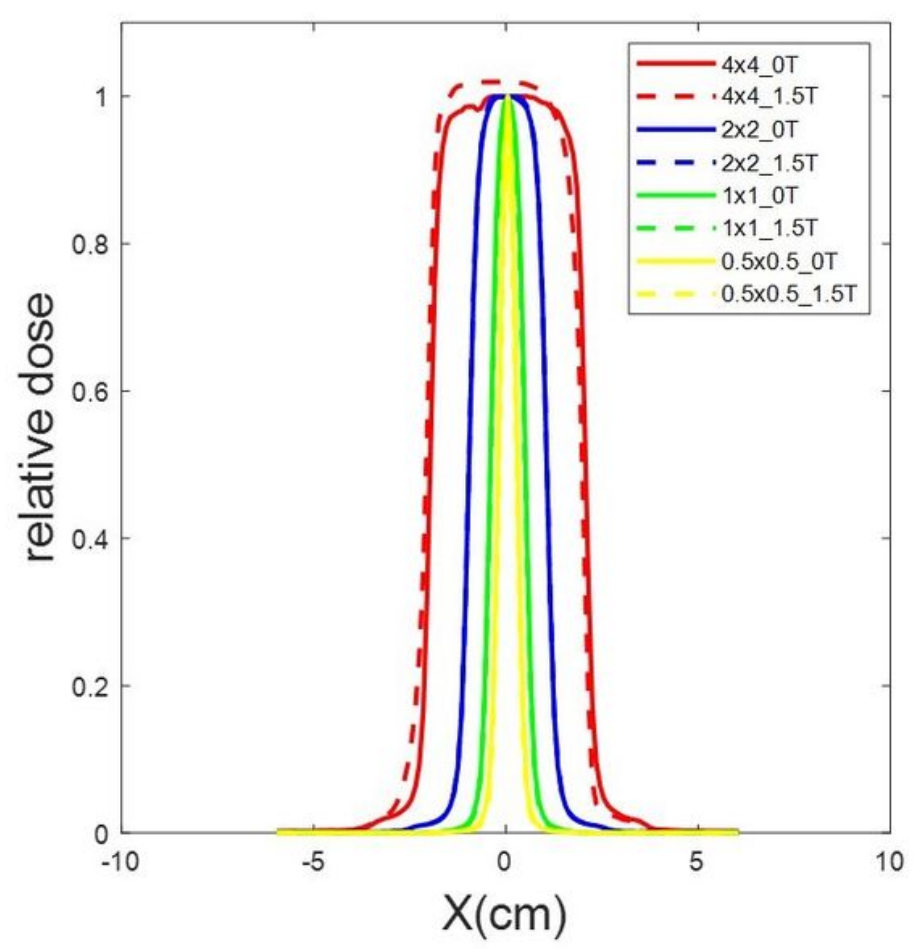

(a)

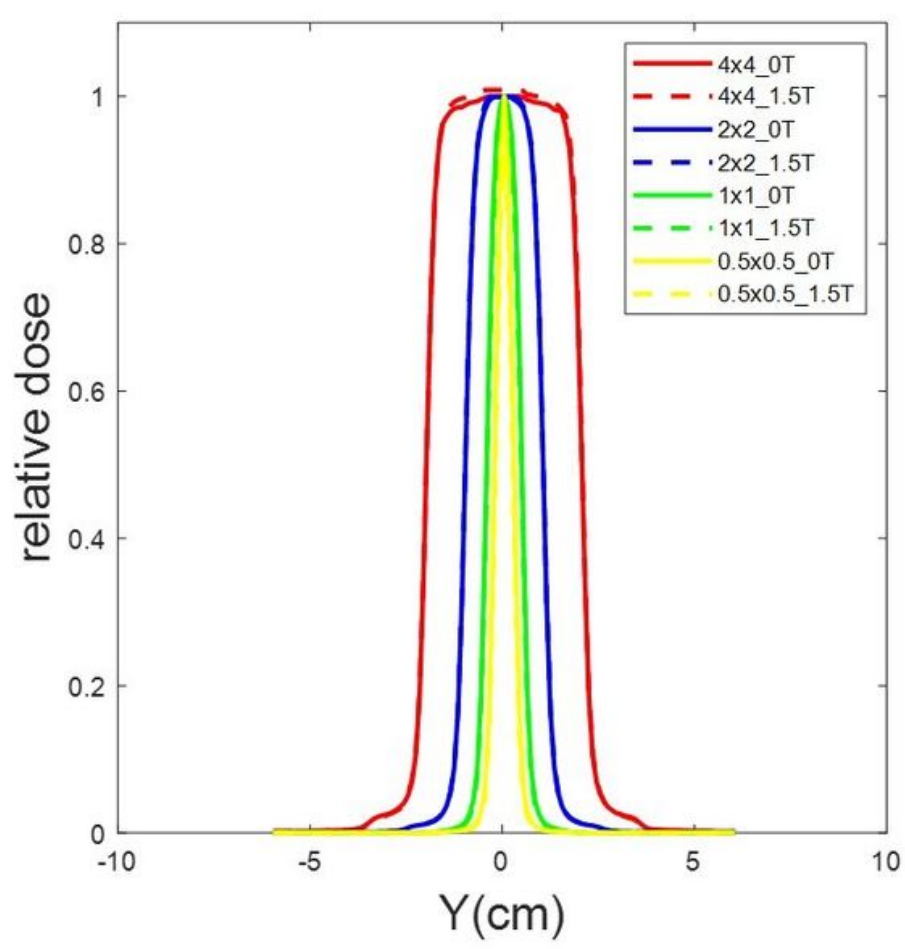

(b)

Figure 3

The dose profiles of water phantom in different size of beam field with magnetic field $B=1.5 T$ compared with those without magnetic field at the depth of the maximum dose. (a) Dose profiles in the $X$ direction. (b) Dose profiles in the $Y$ direction 


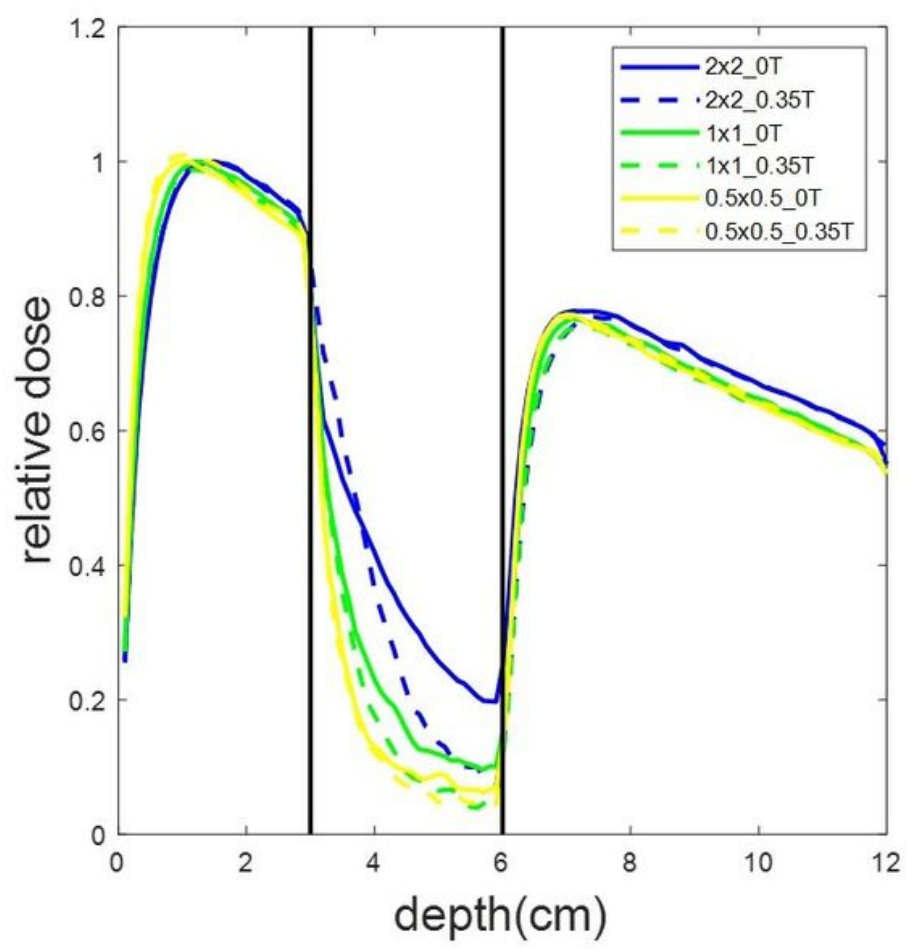

(a)

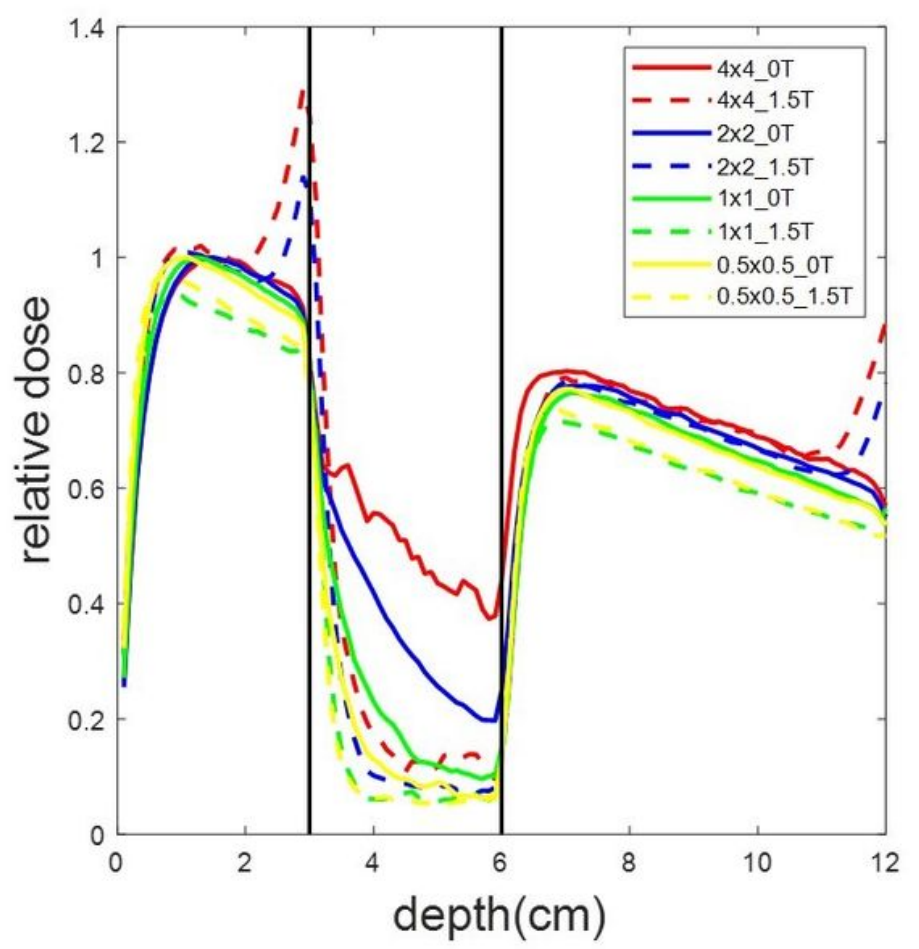

(b)

Figure 4

The PDD curves of water-air phantom in different size of beam field with magnetic field compared with those without magnetic field. (a) $B=0.35 \mathrm{~T}$. (b) $B=1.5 \mathrm{~T}$

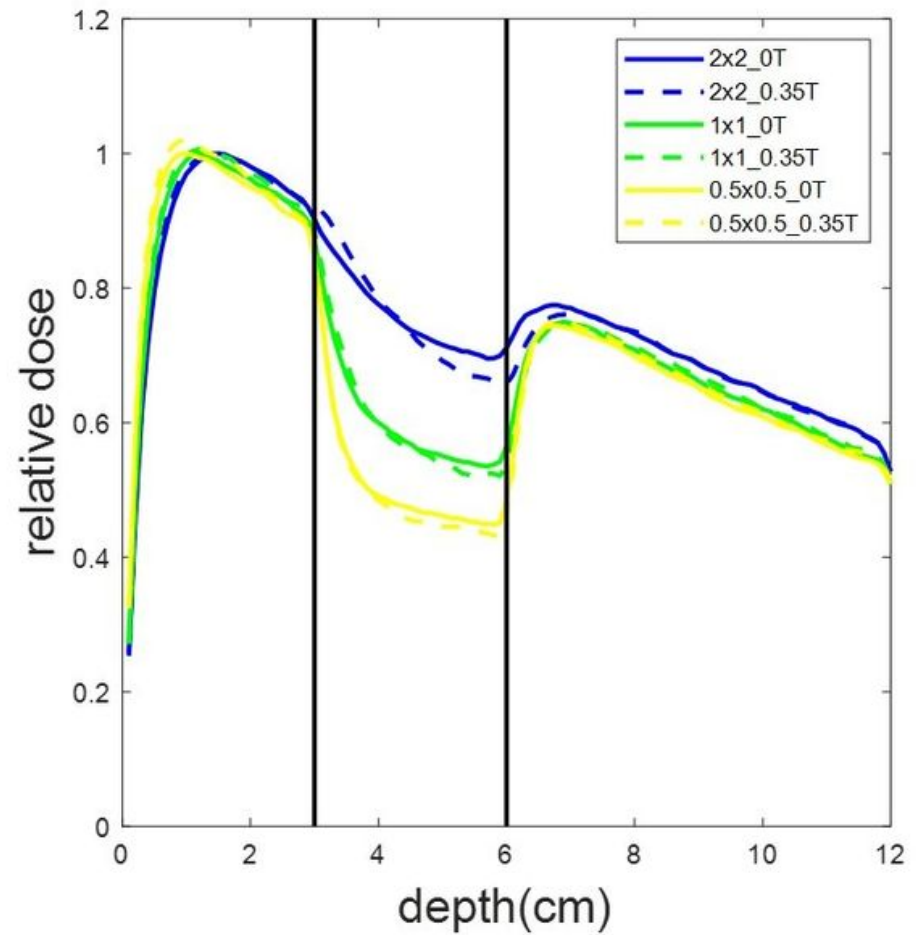

(a)

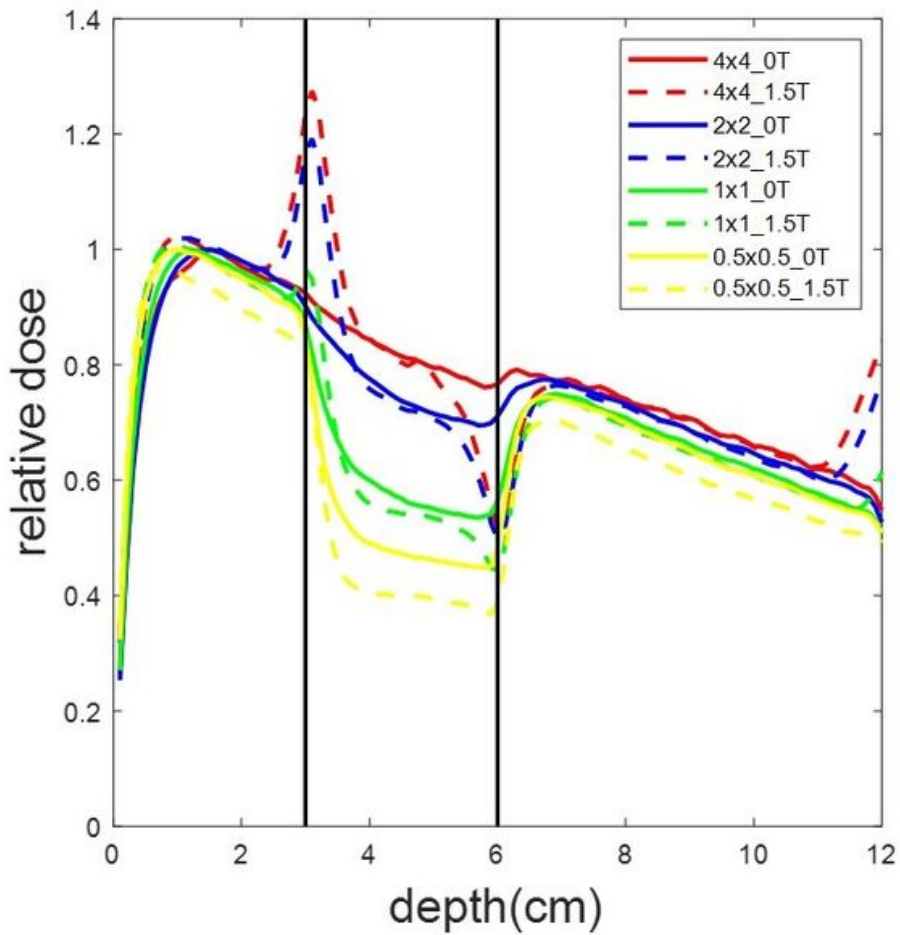

(b) 
Figure 5

The PDD curves of water-lung phantom in different size of beam field with magnetic field compared with those without magnetic field. (a) $B=0.35 \mathrm{~T}$. (b) $B=1.5 \mathrm{~T}$

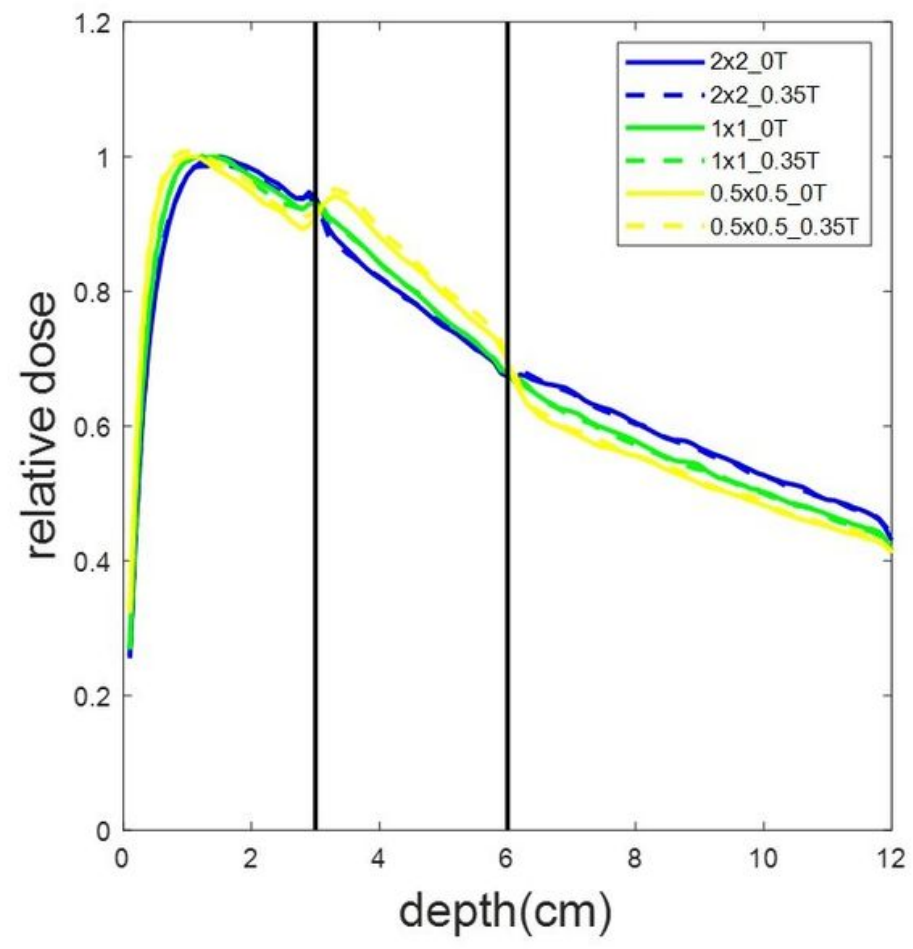

(a)

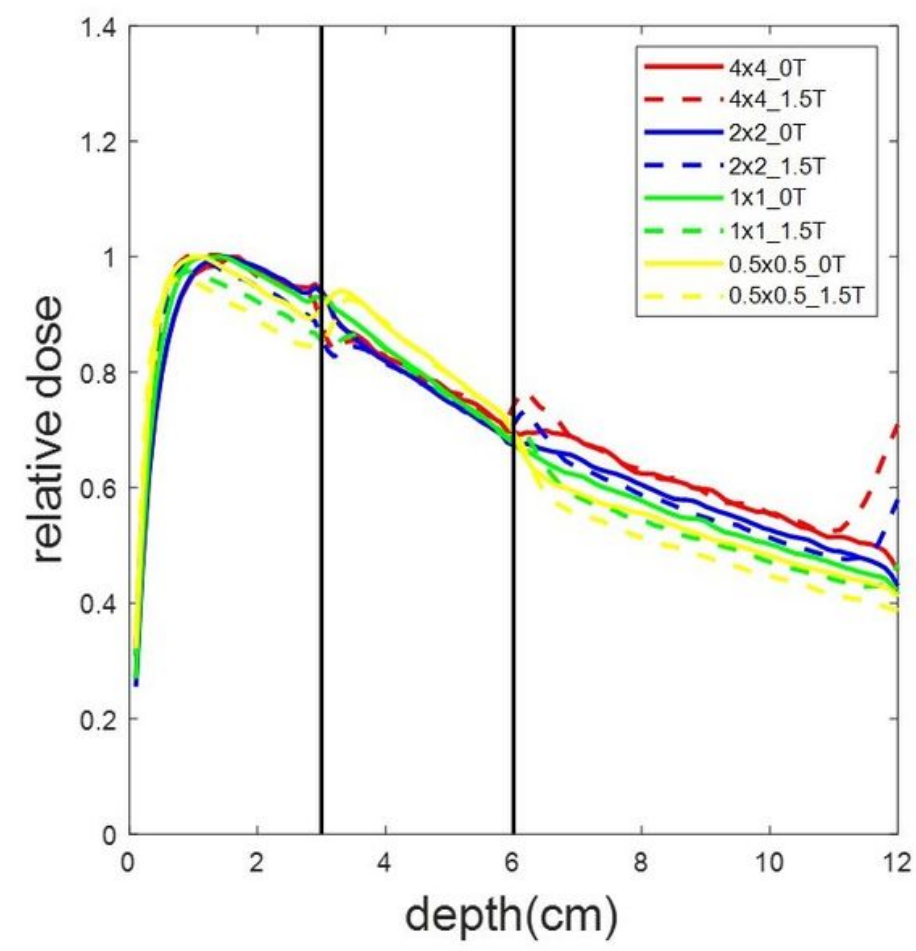

(b)

\section{Figure 6}

The PDD curves of water-bone phantom in different size of beam field with magnetic field compared with those without magnetic field. (a) $B=0.35 \mathrm{~T}$. (b) $B=1.5 \mathrm{~T}$ 